\title{
Self-co-operative ternary pulse-compression sequences
}

\author{
$\mathrm{K} \mathrm{S} \mathrm{RAO}^{1}$ and P S MOHARIR ${ }^{2}$ \\ ${ }^{1}$ Department of Electronics and Communication Engineering, Osmania \\ University, Hyderabad 500 007, India \\ ${ }^{2}$ National Geophysical Research Institute, Uppal Road, Hyderabad 500007 , \\ India
}

MS received 4 May 1996; revised 3 August 1996

\begin{abstract}
An algorithm called a Hamming scan was developed recently for obtaining sequences with large merit factors and is adopted here to obtain such sequences within which there are nontrivial segments of large merit factors. Correlative detection of the return signal can be based simultaneously on the entire sequence and its segments with large merit factors. Such a coincidence detection scheme can be characterized by a Schur merit factor of the sequence. Sequences with large Schur merit factors are listed.
\end{abstract}

Keywords. Self-co-operative sequence; auto-correlation; Hamming scan; Schur merit factor.

\section{Introduction}

The problem of signal design in radar consists of obtaining sequences with prescribed finite alphabet and peaky autocorrelation. The peakiness of the autocorrelation can be characterized by a merit factor (Golay 1977). Three finite alphabets considered in the literature are binary $(+1,-1)$, ternary $(0,-1,+1)$ and quinquenary $(0,+1,-1,+2,-2)$. The largest merit factors obtained so far with these alphabets are 14.0833 (Golay 1977), 20.0556 (Moharir et al 1985; Singh et al 1996; Moharir et al 1996) and 162.000 (Moharir $\&$ Rao 1996). These are achieved for the lengths 13,23 and 7 respectively, which are rather small. As the length increases, it becomes more difficult, on an average, to obtain very high merit factors. However, it is established (Moharir et al 1996; Moharir \& Rao 1996) that the constraint of binary alphabet would have to be overcome if superior merit factors are desired. But this alone may not suffice. Therefore, the notion of complementary or cooperative sequences (Golay 1961; Boehmer 1967; Tseng \& Liu 1972; Venkata Rao et al 1986) has been introduced. These are sets of sequences of which the co-operative merit factor is very high. It can even be infinity. The difficulty is that two or more sequences have to be transmitted and they may fare differently over fading channels. The question, 
therefore, is whether the notion of co-operation can be used, while still having to transmit a single sequence.

The answer to this question is in the affirmative. A notion of towers (Moharir et al 1984) was introduced earlier. A tower was defined to be a sequence with good aperiodic autocorrelation, embedded in which there are marked segments of nontrivial lengths but good aperiodic autocorrelation. This sequence is transmitted and the return signal is crosscorrelated separately with this sequence and its marked segments, maintaining proper temporal relation among them. All the cross-correlations would simultaneously peak at the delay equal to the two-way travel time. Thus, the distance to the target can be estimated on the basis of coincidence of the peaks in various cross-correlations. This notion is extended here in two ways. First, no algorithm was available to list good towers or self-co-operative sequences. Now, an algorithm developed for signal design is adopted for this purpose. Second, a quantitative measure to characterize self-co-operative sequences is proposed.

\section{Earlier signal design algorithms}

Signal design problem can be viewed as an optimization problem (Bernasconi 1987; Golay \& Harris 1990; De Groot et al 1992). One of the effective optimization algorithms for such combinatorial problems is a genetic algorithm (Holland 1975, 1992; De Jong 1985; Michalewicz 1992). But it gives undue importance to chance. An algorithm called eugenic algorithm was developed recently (Singh et al 1996) and used to list ternary sequences with high merit factors. It supplements chance by the notion of a locally complete search. One component of this algorithm is a Hamming scan. Mutation in genetic algorithm is changing one element in the sequence. That is, the result is a first order Hamming neighbour. The Hamming scan looks at all the first order Hamming neighbours and picks up the one with the largest merit factor. The process is recursively repeated as long as the improvement in merit factor continues. Modification of such a recursive Hamming scan is adopted as a procedure in the next section. The next step (Moharir et al 1996) was to use the Kronecker product of two sequences as a starting point for the recursive Hamming scan. This algorithm was called the SIKH algorithm and led to very good long ternary sequences. They are used in the next section for the present purpose.

\section{Design algorithm for self co-operative sequences}

Let

$$
\mathbf{S}_{\mathbf{0}}=\left(s_{0}, s_{1}, \ldots, s_{N-2}, s_{N-1}\right)
$$

be a sequence of length $N$. Let

$$
\mathbf{s}=\left(s_{j}, s_{j+1}, \ldots, s_{j+n-2}, s_{j+n-1}\right)
$$

be a marked segment of length $n$ in it, with $j$ prescribed.

The algorithm takes a sequence $\mathbf{S}_{\mathbf{0}}$ of a large length $N$ obtained by any good algorithm such as the SIKH algorithm (Moharir et al 1996; Moharir \& Rao 1996). This sequence has a good merit factor. Then it embeds the segment $s$ in it at the prescribed place determined 
by $j$. That is, segment $\mathbf{s}$ overwrites the elements, from $j$ to $j+n-1$ in $\mathbf{S}_{\mathbf{0}}$. The segment $\mathbf{s}$ could be a sequence obtained by any good algorithm. This step alters the sequence $\mathbf{S}_{\mathbf{0}}$ to $\mathbf{S}_{\mathrm{a}}$ and brings down its merit factor. Next the sequence $\mathbf{S}_{\mathrm{a}}$ is improved by successive applications of the Hamming scan without touching the elements from $j$ to $j+n-1$. This restricted Hamming scan mutates the elements from 0 to $j-1$ and $j+n$ to $N-1$, one at a time and finds out which among these Hamming neighbours has the largest merit factor. If it is greater than the starting merit factor, the algorithm shifts to that Hamming neighbour. The procedure is continued till the merit factor stops improving. The resultant sequence is $\mathbf{S}$. Thus, the merit factor of the marked segment $\mathbf{s}$ is good because the segment is directly embedded, and the merit factor of the entire sequence $\mathbf{S}$ is good because the recursive restricted Hamming scan has been used for improvement.

\section{Results}

The results are explained first with an example with moderate lengths. The marked segment $\mathbf{s}$ is a ternary sequence of length 23 and has a merit factor of 20.0556 . The length of the sequence $\mathbf{S}_{\mathbf{0}}$ in which it is embedded centrally is 33 . Because $\mathbf{s}$ is embedded centrally, it can be called the core. Before this embedding, the merit factor of the sequence $\mathbf{S}_{\mathbf{0}}$ is 19.5313 . After embedding it falls down but is improved to 5.25 by recursive restricted Hamming scan. There is a precaution to be taken in using this algorithm. The restricted Hamming scan can improve the merit factor by converting most of the elements outside the core to zero, that is, at the cost of the energy efficiency. Therefore, the algorithm should be stopped by ensuring that the energy efficiency does not become too low. We have set up a energy efficiency threshold of 0.6000 .

The merit factor of the core $\mathbf{s}$ is no more a measure of its goodness, as it is embedded in a bigger sequence $\mathbf{S}$ and as it would be cross-correlated with the returned version of $\mathbf{S}$ and not of $\mathbf{s}$ alone. Hence, a quantitative measure of the goodness of the self-co-operative sequence $\mathbf{S}$ with marked core $\mathbf{s}$, proposed is as follows. Let the cross-correlations of $\mathbf{S}$ and $\mathbf{s}$ with the return signal be $\mathbf{C}_{\mathbf{S}}$ and $\mathbf{C}_{\mathbf{s}}$. Let their Schur (component-wise) product be $\mathbf{C}$ and be called Schur correlation. Merit factor $F$ of a sequence $\mathbf{S}$ is defined as the ratio of the energy in the main peak of its aperiodic autocorrelation to the total energy in all its sidelobes. The Schur merit factor SF of the self-co-operative sequence $\mathbf{S}$ with the marked segment $\mathbf{s}$ is defined as the ratio of the energy in the main peak of the Schur correlation $\mathbf{C}$ to the total energy in all its sidelobes. Figures $1 \mathrm{~A}$ and $\mathrm{C}$ show the autocorrelation of $\mathbf{s}$ and $\mathbf{S}$. Figures $1 \mathrm{~B}$ and $\mathrm{D}$ show $\mathbf{C}_{\mathbf{s}}$ (which is different from the autocorrelation of $\mathbf{s}$ ) and $\mathbf{C}$. It can be seen that $\mathbf{C}$ is very peaky. Various lengths and merit factors are indicated on the figure.

Figure 2 is a similar figure, except that the length of the core $\mathbf{s}$ is now 275 and the length of the total sequence $\mathbf{S}$ is 625 . The peakiness of the Schur correlation $\mathbf{C}$ is much more remarkable as both $\mathbf{s}$ and $\mathbf{S}$ are long. In fact, sidelobes are not seen at all. The earlier example was merely to demonstrate the ideas involved at an acceptable horizontal and vertical resolution in the figures.

Table 1 lists the results obtainable with core length of 336. Results not reported here indicate that longer cores are preferable for better results. 

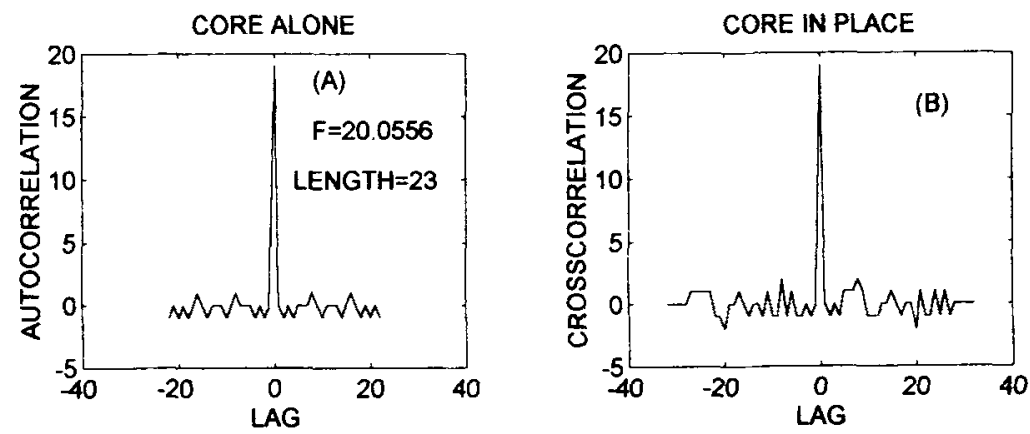

SELF-CO-OPERATIVE SEQUENCE S
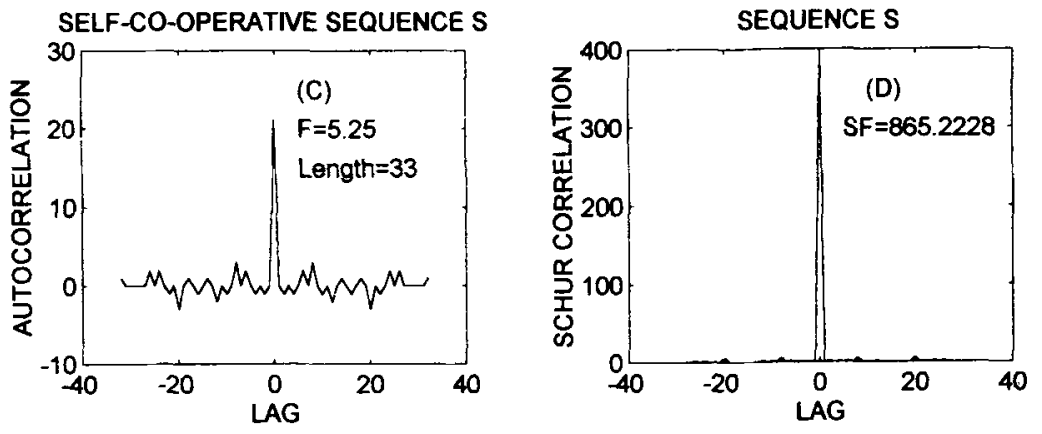

Figure 1. (A) Autocorrelation marked core s. (B) Cross-correlation of the marked core $\mathbf{S}$ with the return version of $\mathbf{S}$. Note that this is different from the autocorrelation of $\mathbf{S}$ and depends on both $\mathbf{s}$ and $\mathbf{S}$. (C) Autocorrelation of the self-co-operative sequence S. (D) Schur correlation of $\mathbf{S}$ and $\mathbf{s}$. Various lengths and merit factors are shown.
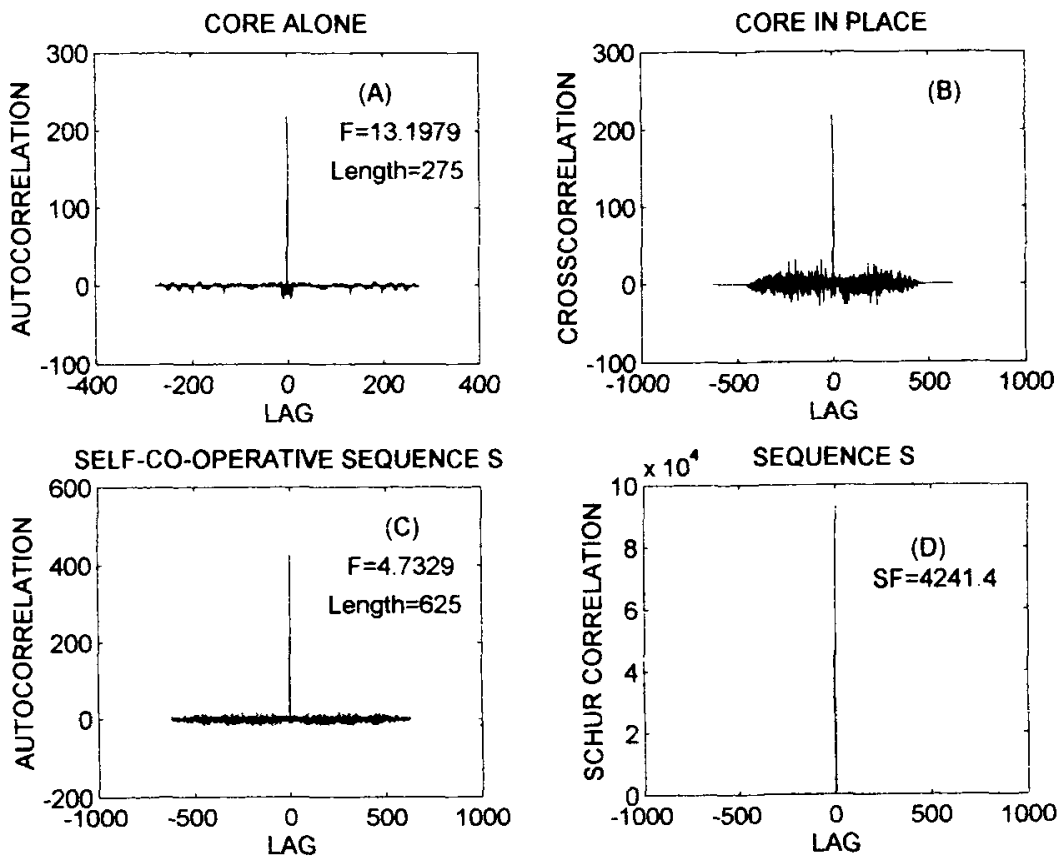

Figure 2. Same as figure 1, except that the lengths of the marked core and the self-co-operative sequence are much higher. 
Table 1. Details of some good self-co-operative sequences with marked core of length 336 .

\begin{tabular}{lccccccc}
\hline \multicolumn{7}{c}{ Merit factor of the core $=10.05$} \\
\hline Length & $\begin{array}{c}\text { Energy } \\
\text { efficiency }\end{array}$ & $\begin{array}{c}\text { Merit } \\
\text { factor }\end{array}$ & $\begin{array}{c}\text { Schur } \\
\text { merit factor }\end{array}$ & Length & $\begin{array}{c}\text { Energy } \\
\text { efficiency }\end{array}$ & $\begin{array}{c}\text { Merit } \\
\text { factor }\end{array}$ & $\begin{array}{c}\text { Schur } \\
\text { merit factor }\end{array}$ \\
\hline 672 & 0.600 & 4.629 & 5166.127 & 728 & 0.636 & 4.693 & 2324.526 \\
736 & 0.609 & 4.652 & 4415.978 & 750 & 0.600 & 4.633 & 4767.170 \\
768 & 0.602 & 4.622 & 4167.685 & 780 & 0.629 & 4.749 & 4673.124 \\
800 & 0.646 & 4.447 & 4121.099 & 806 & 0.614 & 4.480 & 3436.321 \\
816 & 0.643 & 4.580 & 3360.117 & 828 & 0.629 & 4.681 & 4068.622 \\
832 & 0.624 & 4.958 & 4651.332 & 840 & 0.648 & 4.723 & 3654.543 \\
848 & 0.642 & 4.478 & 3716.679 & 858 & 0.619 & 4.981 & 3759.786 \\
868 & 0.624 & 4.851 & 3902.958 & 874 & 0.610 & 4.621 & 3520.839 \\
896 & 0.625 & 4.847 & 3963.063 & 910 & 0.616 & 5.250 & 4400.841 \\
912 & 0.651 & 4.788 & 3585.806 & 924 & 0.603 & 4.845 & 2734.265 \\
928 & 0.655 & 4.792 & 4002.008 & 936 & 0.625 & 5.230 & 4382.247 \\
954 & 0.633 & 4.736 & 3222.210 & 960 & 0.645 & 5.255 & 4235.305 \\
966 & 0.619 & 5.296 & 4814.584 & 972 & 0.640 & 5.253 & 4294.925 \\
980 & 0.612 & 5.566 & 4244.196 & 990 & 0.629 & 5.128 & 3638.130 \\
992 & 0.626 & 5.017 & 4514.279 & 1058 & 0.659 & 5.184 & 3726.345 \\
\hline
\end{tabular}

\section{Conclusion}

The signal design problem can be solved more satisfactorily if part of the burden of obtaining good results can be shared by additional signal processing at the receiver. Here is a scheme in which only one sequence is transmitted and yet the advantages of cooperation are available. Such a coincidence detection scheme would be readily usable. As ternary sequences support better merit factors, they also offer better self-co-operative sequences.

The restricted Hamming scan concept can be readily extended to cover emplacement of more than one marked segment in a sequence. It can also be used recursively in that the self-co-operative sequence $\mathbf{S}$ can be used as a marked segment in a longer self-co-operative sequence. It has been found that a marked core is better in performance than a marked prefix in the self-co-operative sequence.

The authors are grateful to Dr H K Gupta of the National Geophysics Research Institute and Prof R V B Chary of the Osmania University for encouragement and support.

\section{References}

Bernasconi J 1987 Low autocorrelation binary sequences: Statistical mechanics and configuration space analysis. Proc. IEEE 74: 366 
Boehmer M A 1967 Binary pulse compression codes. IEEE Trans. Inf. Theory IT-13: 156-167

De Groot C, Wurtz D. Huffman K H 1992 Low autocorrelation binary sequences: exact enumeration and optimization by evolutionary strategies. Optimization 23 : 369-384

De Jong K A 1985 Genetic algorithms: a 10 year perspective. In Proc. First Int. Conference on Genetic Algorithms (ed.) J J Grefenstette (Hillsdale: Lawrence Erlbaum) pp 169-177

Golay M J E 1961 Complementary series. IEEE Trans. Inf. Theory IT-7: 82-87

Golay M J E 1977 Sieves for low autocorrelation binary sequences. IEEE Trans. Inf. Theory IT-23: 43-5:

Golay M J E, Harris D 1990 A new search for skew-symmetric binary sequences with optimal merit factors. IEEE Trans. Inf. Theory 36: 1163-1166

Holland J H 1975 Adaptation in natural and artificial systems (Ann Arbor: University of Michigan Press)

Holland J H 1992 Genetic algorithms. Sci.Am. 267: 66-72

Michalewicz Z 1992 Genetic algorithms + data structures = evolution programs. (Berlin: Springer-Verlag) p. 255

Moharir P S and Rao K S 1996 Nonbinary sequences with superior merit factors (submitted)

Moharir P S, Venkata Rao K, Varma S K 1984 Barker towers. Electron. Lett. 20: 1027-1029

Moharir P S, Varma S K, Venkata Rao K 1985 Ternary pulse compression sequences. J. Inst. Electron. Telecommun. Eng. 31:33-40

Moharir P S, Singh R, Maru V M 1996 S-K-H algorithm for signal design. Electron. Lett. 32: $1648-1649$

Singh R, Moharir P S, Maru V M 1996 Eugenic algorithm-based search for ternary pulse compression sequences. J. Inst. Electron. Telecommun. Eng. 42: 11-19

Tseng C C, Liu C L 1972 Complementary sets of sequences. IEEE Trans. Inf. Theory IT-18: 644-652

Venkata Rao K, Moharir P S, Varma S K 1986 Doubly co-operative ternary sequences. Proc. Inst. Elecr. Eng. F133: 61-67 